\title{
Software Maintenance
}

National Cancer Institute

\section{Source}

National Cancer Institute. Software Maintenance. NCI Thesaurus. Code C67227.

All program coding after initial deployment to correct coding errors, enhance the functionality of the software, and perfect the functioning of the software. 